\title{
NON-DISJUNCTION IN AN ENOTHERA INTERCHANGE HETEROZYGOTE
}

\author{
D. G. CATCHESIDE \\ Department of Microbiology, University of Birmingham
}

\section{INTRODUCTION}

Received 15.vi.62

THIs paper was first drafted about ten years ago, but not submitted for publication (though predicted as Catcheside, I953 in Catcheside, I954) because some of the possible kinds of plants had not been observed, leaving the web of evidence incomplete. In the interval, very little has been done to fill the gaps. Some discussions (e.g. Goldschmidt, I958) have relied on an incorrect interpretation made previously (Catcheside, $19476)$ and it is therefore considered that the paper, though it may be deficient in certain particulars, should nevertheless appear.

Before dealing with the exceptional plants, it may be useful to recount the genetics of the position effect to which they are related. In Enothera blandina, ir radiation of pollen gave a number of chromosomal interchanges, one of which, designated blandina- $A$, resulted in marked differences in habit and foliage characters and in variegation effects on certain genes, notably $P^{r}, P^{s}$ and $S$ which are in chromosome arm 3 . The ${ }^{h}$ blandina chromosomes concerned in the interchange are 3.4 and II.12, the blandina- $A$ chromosomes being $3 \cdot I_{1}$ and $4 \cdot 12$, defined according to the standard system generally accepted (Catcheside, 1940). The segments exchanged are unequal in size, so that the interchange chromosomes are recognisable individually. In an interchange heterozygote, having either $P^{r}$ or $P^{s}$ on the $3 \cdot 1$ I chromosome of blandina-A and $P^{s}$ on the 3.4 chromosome of hblandina, the sepals are variegated, respectively with patches of dark $\left(P^{r e}\right)$ or medium $\left(P^{s e}\right)$ red on a green background. If $P^{r}$, or $P^{s}$, is removed from $3 \cdot$ I I to 3.4 by crossing over, its property of full colour is recovered. A similar variegation is shown by the petal colour gene $S$ when it is on the interchange chromosome $3 \cdot \mathrm{I}$, but not when it is in the normal chromosome 3.4 . Thus in the cross between the heterozygote $P^{r}$ blandina- $A P^{s}$ hblandina, which shows $P^{r}$ variegation $\left(P^{r e}\right)$, and normal blandina ( $P^{s}$ hblandina $/ P^{s}$ hblandina), four types of regular progeny are cxpected. Two are like the respective parents and two are recombinant types, the latter constituting about I per cent. of the progeny. The expected recombinants are $P^{s}$ blandina- $A / P^{s}$ blandina, which shows $P^{s}$ variegation $\left(P^{s e}\right)$, and $P^{r}$ hblandinal $P^{s}$ hblandina, which shows deep red (rubricalyx) buds.

\section{(i) Nature of duplication and deficiency plants}

In a previous paper (Catcheside, 1947b) two types of exceptional progeny were discussed. They were shown to result, respectively, 
from a duplication $(A-D P)$ and a deficiency $(A-D f)$ of the $P$-s region of chromosome arm 3 in gametes formed by the interchange heterozygote. It was concluded that the exceptional gametes possessed chromosomes $3 \cdot 11+4 \cdot 12$, like the interchange gamete blandina- $A$, except that the 3.11 chromosome of $A-D p$ had a direct contiguous duplication of the $P$-s region, while the 3 .I I chromosome of $A$ - $D f$ lacked this region altogether. However, this interpretation could not be regarded as proved beyond doubt and further investigation has shown it to be wrong. The correct interpretation, that $A-D p$ and $A-D f$ represent two
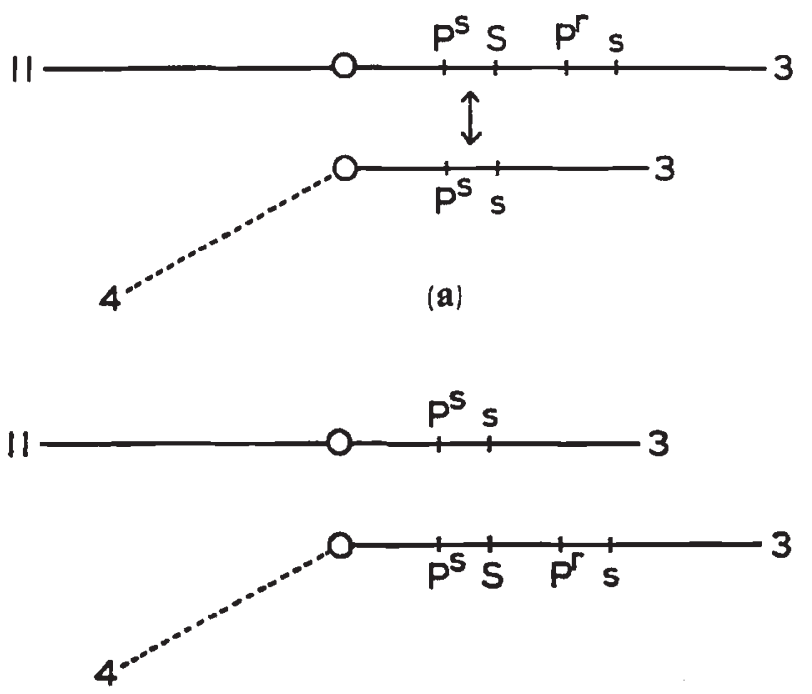

(b)

FIG. 1.-Diagrams of pairing relationships on the assumption of duplication within the chromosome $3 \cdot 1$ in $A-D p$ plants.

of the non-disjunctional types of gametes which originate from the interchange heterozygote, was previously disregarded mainly on the naive assumption that they would be inviable.

If the hypothesis of duplication within the $3 \cdot$ I I chromosome were correct, it should be possible to recover the original blandina- $A$ from $A-D p$. In the previous work, it was believed that this had been shown (Catcheside, 1947b, table $3 a$ and page 107). However, at that time it was thought that $A-D p$ plants were " not distinguishable by any conspicuous characters of habit or foliage from corresponding plants lacking the duplication (referred to as $A$ ). There are slight differences, but it is doubtful whether they allow a reliable diagnosis." Since then, further experience has enabled the recognition of the two types, without any doubt, on the basis of habit and foliage characters, independently of the bud characters which are associated with the position effect expression and which depend upon the particular genes present in interchange chromosomes and their dosages. About 1126 
progeny have been grown from $A-D p$ plants and no blandina- $A$ individuals have been found amongst them. The search has been made more definitive by marking the $A-D p$ chromosomes so that the number of cross overs, presumably complementary to the blandina- $A$ individuals sought for, could be determined.

First, $A-D p$ plants were obtained from blandina- $A P^{s} S /{ }^{h} b l a n d i n a$ $P^{r}$ by pollinating with blandina $P^{s}$ plants. On the hypothesis of a

TABLE I

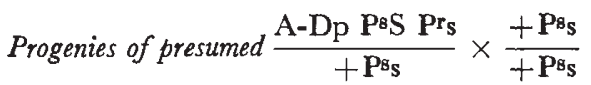

\begin{tabular}{|c|c|c|c|c|c|}
\hline \multirow{2}{*}{ Phenotype } & \multicolumn{4}{|c|}{ Family } & \multirow{2}{*}{ Totals } \\
\hline & $\frac{50}{50}$ & $\frac{5 I}{50}$ & $\frac{52}{50}$ & $\frac{6 \text { families }}{44}$ & \\
\hline $\begin{array}{l}+P^{r} S \\
+P^{r} s \\
+P^{s} S \\
+P^{s} s\end{array}$ & $\begin{array}{r}2 \\
33 \\
1 \\
31\end{array}$ & $\begin{array}{r}3 \\
65 \\
5 \\
38\end{array}$ & $\begin{array}{r}3 \\
24 \\
4 \\
36\end{array}$ & $\begin{array}{r}6 \\
5 ! \\
8 \\
62\end{array}$ & $\begin{array}{r}14 \\
173 \\
18 \\
167\end{array}$ \\
\hline $\begin{array}{l}A-D p P^{r} S^{e} \\
A-D p P^{r} s \\
A-D p P^{r e r_{s}} \\
A-D p P^{s} S^{e} \\
A-D p P^{s_{s}} .\end{array}$ & 6 & $\begin{array}{r}\text { II } \\
\text { I } \\
\text { I } \\
9 \\
\text { I }\end{array}$ & 10 & 16 & $\begin{array}{r}33 \\
1 \\
1 \\
32 \\
1\end{array}$ \\
\hline Totals & 75 & I 34 & 81 & 150 & 440 \\
\hline
\end{tabular}

Symbol

Phenotype

$P^{s}$ medium red buds, with fine green stripes on ribs and margins of sepals.

$P r$ deep red, uniformly coloured buds.

Prer variegated deep red buds, with a medium red background.

$S$ yellow petals.

$s$ sulphur petals.

$S^{*}$ variegated (yellow and sulphur) petals.

tandem duplication within the 3 . I I chromosome in $A-D p$, this chromosome would have the structure II $\cdot P^{s} S P^{r_{S}} 3$, which is depicted in fig. I. With $P_{s}$ in the 3.4 chromosome, the bud and petal colour phenotypes of the $A-D p$ plants would be fully red bud colour (rubricalyx) and variegated, yellow and sulphur, petal colour. The $A-D p$ plants could produce blandina- $A$ gametes if the $P^{s} S$ section of the $A-D P 3 \cdot$. I chromosome paired and crossed over with the $P^{s}$ s section of the blandina 3.4 chromosome (fig. I a). Irrespective of whether crossing over occurred in the region between the centromeres and the $P$ locus or in the region between the $P$ and $s$ loci, the blandina 3.4 chromosome resulting should have the composition $P^{s} S P^{r} s$, while the blandina- $A$ $3 \cdot$ II chromosome should have the composition $P^{s_{s}}$. The blandina 
progeny would have the phenotype $P^{r} S$, that is have deep red bud colour (rubricalyx) and yellow petals. The blandina- $A$ progeny would have the phenotype $P^{s e}$, that is have light red, variegated bud colour and sulphur petals.

The available progenies are classified in table $\mathrm{I}$, omitting one haploid plant and also 2 I blandina plants and $9 A-D p$ plants which failed to flower and so could not be scored completely. It will be noted that $S$ has crossed over from the 3.1 i chromosome to the 3.4 chromosome fourteen times to give $P^{r} S$ plants. The complementary cross over, appearing as $P$ se blandina- $A$ plants, should have occurred about the same number of times amongst the $44^{\circ}$ progeny plants observed. Thus each plant in the progenies should have a chance $\frac{-14}{440}$ of being the type sought. It appears, then, that the chance of obtaining none of them amongst 440 plants is $\left(\frac{42 \frac{6}{4}}{44}\right)^{440}$ or about $\mathrm{I} \cdot 8 \times 1 \mathrm{IO}^{-6}$. Even the known lesser viability of blandina- $A$ could not account for very much of the improbability of this result. It must be concluded that the previous hypothesis of the particular structure of the $A-D p P s$ duplication is wrong. A different form of duplication must be considered, taking into account the evidence that the $A-D p$ plants do not contain all the elements required to segregate out a blandina- $A$ gamete.

\section{(ii) Cytological evidence}

The blandina- $A$ interchange, between chromosomes 3.4 and II 12 , involves an exchange of segments of unequal size. Whereas chromosomes 3.4 and II 12 are of similar size, each with a median centromere, chromosomes $3 \cdot 11$ and 4.12 are unequal in size, one having an arm relatively longer and the other an arm relatively shorter than the lengths characteristic of the arms of 3.4 and II 12 . It will be shown, later in this paper, that the longer chromosome is 3.11 and the shorter one is $4 \cdot 12$.

Improved methods of preparing slides of the meiotic chromosomes have disclosed further details. The most satisfactory fixative is Bradley's mixture, namely three volumes of absolute alcohol, one volume of glacial acetic acid and four volumes of chloroform, freshly mixed. Squash preparations, stained with iron aceto-carmine, are made in the usual way. These disclose differential staining of different parts of the chromosomes, visible clearly at diakinesis and sometimes even at metaphase I. All of the chromosomes of $O$. blandina, like the bivalents in figure $2 a$, have a relatively large, deeply stainable segment which includes the centromere, and two smaller less stainable segments which form the more distal parts of the chromosome arms. The more deeply stainable proximal segments have the characters of being heterochromatic, while the more distal segments are euchromatic. The heterochromatic segments are likewise relatively over-condensed and deeply stainable earlier in the meiotic prophase and perhaps at various stages of the mitotic cycle. The long chromosome of blandina- $A$ (fig. 2a) has, in its long arm, a normal sized heterochromatic segment 
followed, distally, by a short euchromatic segment, then a short heterochromatic segment and, finally, a terminal euchromatic segment of normal size. The short chromosome of blandina- $A$ has, in its short arm, an abbreviated heterochromatic segment followed, distally, by an abbreviated euchromatic segment. The unequal interchange therefore has resulted from breakage in the heterochromatic segment in one chromosome and in the euchromatic segment in the other.

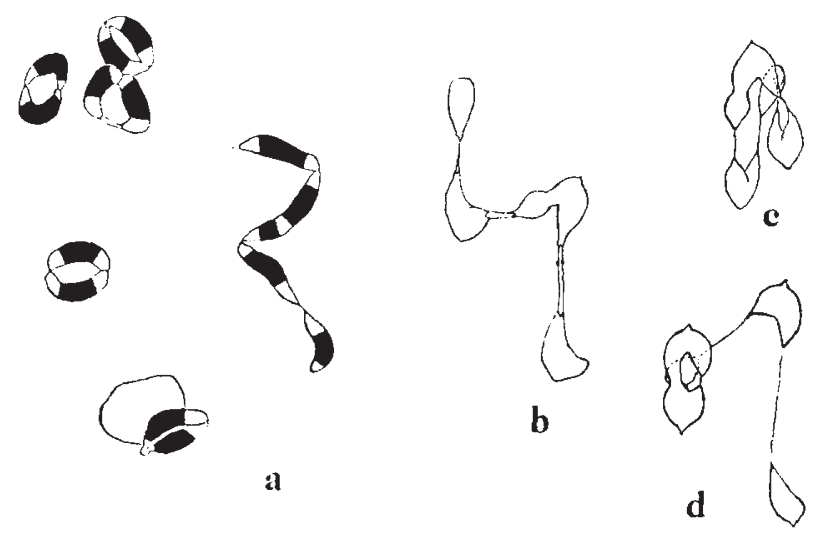

FiG. 2.-Diakinesis (a) and metaphase $\mathrm{I}$ (b-d) in blandina-A. The shaded regions of the chromosomes at diakinesis are heterochromatic.

The $A-D p$ plants show at meiosis (fig. $3 a$ ) an association of four chromosomes, consisting of the long one, with two heterochromatic and three euchromatic segments and three others which appear like normal $O$. blandina chromosomes. They lack the short chromosome. At first

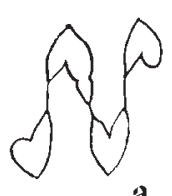

a

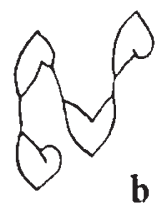

b

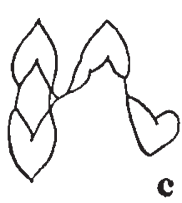

c

FrG. 3.-Meiotic associations in duplication and deficiency plants ; (a) and (c) $A-D P$; (b) $A-D f$.

it was thought that the short chromosome was $3 \cdot$ I I and that one of the chromosomes which appeared normal in size was this chromosome with the duplication. However, the $A-D f$ plants possess the short chromosome together with three normal looking chromosomes making up the association of four (fig. $3^{b}$ ). If the short chromosome were $3 \cdot \mathrm{I}$, the $A-D f$ plants ought to have a still shorter chromosome and also have the long chromosome with the two heterochromatic segments. This expectation is not realised. By no interpretation along these lines can the chromosome sets in the $A-D p$ and $A-d f$ plants be reconciled with one another. 
In the $A-D p$ plants, the association of four chromosomes is most generally a chain (fig. $3 a$ ), in which the long chromosome has two chromosomes of normal size attached to its longer arm and one chromosome of normal size attached to its shorter arm which is really of normal size. Less commonly, the association of four is replaced by a ring bivalent and a rod bivalent, the latter including the long chromosome attached by its normal arm to a chromosome of normal size. In a very few cases, the association of four is of the ring and rod, or " frying pan" type (fig. $3 c$ ) in which the long chromosome is attached by its longer arm to a ring of two chromosomes.

In the $A-D f$ plants, corresponding associations may be seen, with the short chromosome of blandina- $A$ substituting for the long chromosome in corresponding attitudes with respect to the normal chromosomes. That is, similar configurations to those in the $A-D p$ plants would

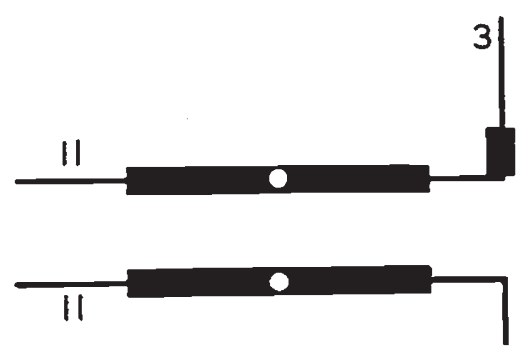

12
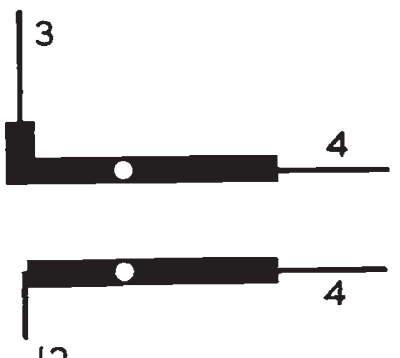

12

FIG. 4.-Diagram of the homologies of the normal ( 3.4 and $I 1 \cdot 12)$ and interchange chromosomes $(3.11$ and 4.12$)$ in the ${ }^{h}$ blandina/blandina-A heterozygote. The heterochromatic regions are shown thicker than the euchromatic ones.

be completely described by substituting "shorter arm" for "longer arm" in each case. However, the relative frequencies are different. The "frying pan" type of association of four has been seen only once, and the chain of four (fig. $3 b$ ) is much less frequent than the ring bivalent plus rod bivalent.

Each of the exceptional gametes certainly has two chromosomes capable of associating with the $3 \cdot 4$ and II I $_{2}$ chromosomes of the bblandina gamete. The $A-D p$ gamete has the long chromosome and the $A-D f$ the short chromosome of the blandina- $A$ gamete. In addition each has a chromosome which is similar in size and other morphological characters to the chromosomes of hblandina. The observations at metaphase I of meiosis show that these normal looking chromosomes, respectively of $A-D p$ and $A-D f$ gametes, are each homologous with a chromosome, either 3.4 or I I 12 , of bblandina. Further, the normal looking chromosome of $A-D p$ is homologous at one end with the long arm of the interchange chromosome, while the normal looking chromosome of $A-D f$ is homologous at one end with the short arm of the short interchange chromosome.

These conclusions imply that $A-D p$ and $A-D f$ are each formed by adjacent non-disjunctional segregation of the ring or chain of four 
chromosomes formed at meiosis by the ${ }^{h}$ blandina/blandina- $A$ interchange heterozygote. Now, it is known from genetical evidence that the $A-D p$ gamete is duplicate for the $P$-s region. It is also known that this region is in arm 3 of chromosome 3.4 . Therefore the long interchange chromosome is 3.1 I and the duplication of the $P-s$ region in $A-D p$ gamete is accounted for by it possessing 3.4 and 3.11 . These two chromosomes are homologous at the 3 end, hence the 3 segment is the distal part of the long arm of the long interchange chromosome.

This conclusion is in agreement with that which may be derived from the $A-D f$ gamete. This gamete is totally deficient for the $P-s$ region and so cannot have either of the chromosomes 3.4 and 3. II which carry this region, but instead must have $4 \cdot I_{2}$ and II I 2 . These two chromosomes are homologous at the 12 end, consequently the distal part of the short arm of the short interchange chromosome is the distal part of the 12 segment. The interchange relations diagrammed in figure 4 are now fully established.

\section{(iii) Gamete production by the interchange heterozygote}

In respect of the four chromosomes, $3 \cdot 4+4 \cdot 12+12 \cdot 11+11 \cdot 3$, making up the association in the interchange heterozygote, gametes containing two or three of these chromosomes may be constituted in ten ways. First, there are the two types of balanced gamete, $3 \cdot 4+\mathrm{I}$ I $\cdot 12$ and $3 \cdot 11+4 \cdot 12$, which are respectively the normal ${ }^{h}$ blandina and blandina$A$ constitutions. These arise by zigzag, nonadjacent co-orientation of the chromosomes at metaphase I of meiosis, so that alternate chromosomes in the ring, or chain, pass to the same spindle pole at anaphase $\mathrm{I}$, adjacent chromosomes passing to opposite poles.

Second, there are the four types of unbalanced gamete, each with two adjacent chromosomes of the four in the interchange association. These are respectively $3 \cdot 4+4 \cdot 12,4 \cdot 12+I 1 \cdot I_{2}$ (which are in $A-D f$ ), II I $2+I I \cdot 3$ and $3 \cdot I I+3 \cdot 4$ (which are in $A-D p$ ). They arise by parallel co-orientation of the ring of four chromosomes at metaphase I and so the passage of two adjacent chromosomes to the same spindle pole at anaphase I. The products of the first and third of these nondisjunctional gametes have not been recognised definitely so far, but will be considered in a later section of this paper.

Third, there are the four types of unbalanced gamete, each with three of the four chromosomes of the interchange association. These are respectively $3 \cdot 4+4 \cdot I 2+I I \cdot I 2,4 \cdot I 2+I I \cdot I 2+3 \cdot I I, I I \cdot I 2+3 \cdot I I$ $+3 \cdot 4$ and $3 \cdot 11+3 \cdot 4+4 \cdot 12$. They arise by the passage of three chromosomes, naturally adjacent in the ring, to the same spindle pole at anaphase I. Combined with a normal gamete $(3 \cdot 4+11 \cdot 12)$, each would yield a trisomic zygote. Thus the first would give the tertiary trisomic subwhitish, the second would give the golden trisomic and the fourth the whitish trisomic (Catcheside, I954), both the latter in a blandina- $A$ heterozygote. The product of the third extra chromosome gamete has not yet been definitely observed; it would be a tertiary 
trisomic and may be expected to resemble the golden trisomic phenotypically. The complementary types of gamete, each with only one of the four chromosomes of the interchange association, theoretically originating when the unbalanced gametes with extra chromosomes are formed, are presumed to be completely inviable as they have not so far been observed. However, the possibility of occurrence of the various sorts of monosomic plants which are possible in progenies of interchange heterozygotes must be kept in mind.

Predictions as to the frequencies with which these ten types of gamete, with two or three of the chromosomes $3.4,3 \cdot 11,4^{\cdot} \cdot 2$ and

\begin{tabular}{|c|c|c|c|c|c|c|}
\hline $\begin{array}{l}\text { Config- } \\
\text { uration }\end{array}$ & 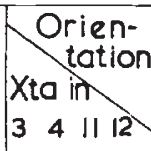 & Alternate & 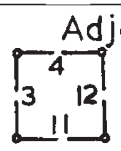 & $\int_{2}^{2 n t}$ & Indifferent & Totals \\
\hline Ring & 1111 & 55 & & 2 & 1 & 58 \\
\hline \multirow{4}{*}{ Chain } & 1110 & 160 & 1 & 5 & 3 & 169 \\
\hline & 1101 & 2 & 1 & & & 3 \\
\hline & 1011 & 4 & & & & 4 \\
\hline & 01111 & 6 & & & & 6 \\
\hline $\begin{array}{l}\text { Two Rod } \\
\text { Bivalents }\end{array}$ & 0110 & 3 & & 2 & & 5 \\
\hline $\begin{array}{l}\text { One Rod Biva- } \\
\text { lent Two } \\
\text { Univalents }\end{array}$ & 0100 & & & & 1 & 1 \\
\hline & $\begin{array}{cccc}1 & 1 & 1 & 0 \\
8 & \text { Int. Segt }\end{array}$ & 2 & & & 1 & 3 \\
\hline & Totals & 232 & 2 & 9 & 6 & 249 \\
\hline
\end{tabular}

FIG. 5.-Types and frequencies of disjunction of the ring of four chromosones observed in $O$. blandina- $A$.

II $\cdot$ I2, originate may be made from observations upon the chromosome configurations and their orientations at metaphase I of meiosis. The available data are summarised in figure 5 and may be compared with the progeny data in table 2 . These data indicate that non-disjunction is quite low in frequency and, by comparison with the relative frequencies amongst recovered zygotes, that several of the nondisjunctional types of gamete have, on the whole, a fairly good chance of functioning. Clearly blandina- $A$ is less viable than ${ }^{b}$ blandina. The relative viabilities of the ten types of gamete may be expressed (table 2) in terms of ${ }^{h} b l a n d i n a$ as unity. The calculation, for $A-D p$ as an example, is simply the division of the ratio of $A-D p$ zygotes to blandina zygotes observed, by the ratio of $3 \cdot 4+3 \cdot I_{1}$ gametes to $3 \cdot 4+$ II ${ }^{2} 2$ gametes inferred from cytological observations. Thus, if the frequencies 
NON-DISJUNCTION IN ENOTHERA

\begin{tabular}{|c|c|c|c|c|c|}
\hline & 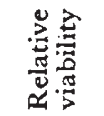 & $\begin{aligned} & \% \\
&-0\end{aligned}$ & $\ddot{0}$ & & \\
\hline & 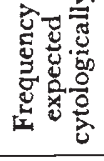 & $\begin{array}{l}\dot{q}+\dot{q} \\
\dot{\phi}\end{array}$ & 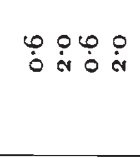 & 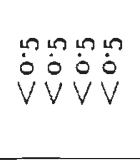 & \\
\hline & 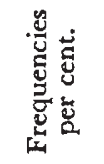 & 商 & 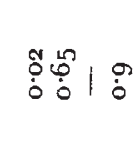 & 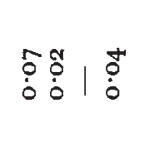 & \\
\hline & 吾 & लैई & आ⿻ & $m=0 \mathrm{~N}$ & $\stackrel{0}{4}$ \\
\hline 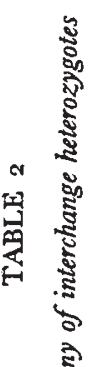 & 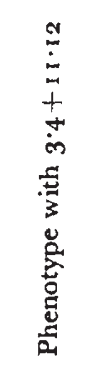 & 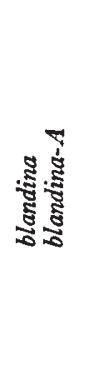 & \} $&{\text { 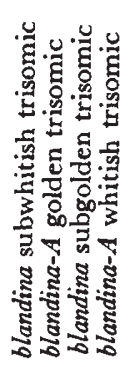 }} &{ } \\
{\hline} &{\text { 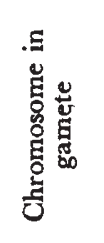 }} &{\text { 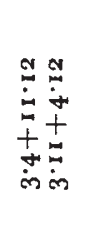 }} &{\text { 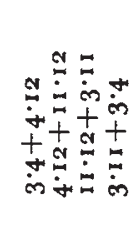 }} &{\text { 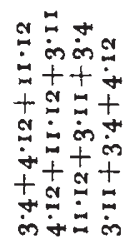 }} &{ } \\
{\hline} &{ } &{\text { 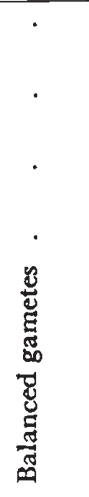 }} &{\text { 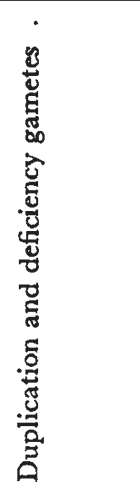 }} &{\text { 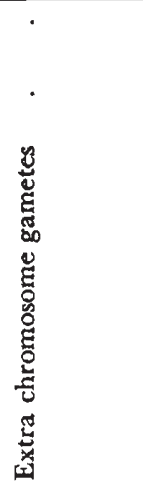 }} &{ } \\
$\hline
\end{tabular}


predicted cytologically are $x$ of blandina and $y$ of blandina- $A D p$, while the plants observed are $m$ of blandina and $n$ of blandina- $A D p$, the relative viability is $\frac{n x}{m y}$ or $\frac{0.9 \times 50 \cdot \mathrm{I}}{46 \cdot \mathrm{I} \times 2 \cdot 0}$ or 0.42 . This expresses the relative chance that an unbalanced female gamete, having been formed in an ovule, will form a viable plant. It assumes that the cytological situation is the same in megaspore mother cells as in pollen mother cells.

These same cytological data may be used to measure chiasma frequencies at metaphase I (table 3 ) between the end pairing segments, since every one of the segments may be separately recognised by reason of the unequal sized chromosomes which occupy fixed positions. The frequencies in segments 3,4 and I I are not significantly different from one another or from what the values are in structural homozygotes. But the frequency in segment 12 is greatly reduced, to about a third of the normal, and the loss is not accountable by chiasmata formed in the interstitial segment common to chromosomes 3 . II and II I 2.

TABLE 3

\begin{tabular}{|c|c|c|c|c|}
\hline Segment & 3 & 4 & II & I2 \\
\hline Chiasma frequency & 0.96 & 0.98 & 0.99 & 0.33 \\
\hline
\end{tabular}

A careful search has been made for the occurrence of chiasmata in the interstitial segments of the interchange heterozygote. A few have been observed (fig. $2 d$ ) but the frequency of the occurrence is very low, only about one per cent. of pollen mother cells showing them. This tends to show that chiasma formation in the proximal part of the chromosome is rare.

\section{(iv) Progeny of A-Dp and A-Df plants}

Each of these kinds of plants is constituted of a normal set of

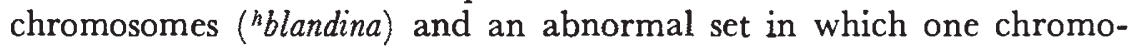
some segment is duplicated and another segment is deficient. Thus $A$ - $D p$ has two 3.4 chromosomes and one each of 3.1 I and I I.I2, while $A$-Df has two II.I2 and one each of 3.4 and $4 \cdot{ }^{12}$. At meiosis, these groups of four chromosomes most commonly form a chain of four chromosomes or a ring and a rod bivalent, but occasionally the ring and rod type of association of four chromosomes, shown in fig $3 c$, occurs and demonstrates the homologies.

The types of gametes likely to be formed by these plants are very restricted in number compared with the interchange heterozygote. Taking $A-D p$ as an example, theoretically four sorts of gametes with two each of the chromosomes $3.4,3.4,3$. I I and II I 2 could occur, namely $3 \cdot 4+3 \cdot I \mathrm{I}, 3 \cdot 4+\mathrm{II} \cdot \mathrm{I} 2,3 \cdot 4+3 \cdot 4$ and $3 \cdot \mathrm{I} I+I \mathrm{I} \cdot \mathrm{I} 2$. However, the last two are most unlikely as each would involve the equivalent of 
a primary non-disjunction. Further, theoretically, three sorts of gametes with three each of these chromosomes could occur, namely the likely one with $3 \cdot 4+3 \cdot 11+11 \cdot 12$, and the unlikely two with $3 \cdot 4+3 \cdot 4$ $+\mathrm{II} \cdot 12$ or $3.4+3.4+3.1 \mathrm{I}$, the latter two involving a primary nondisjunction of the two homologous 3.4 chromosomes.

The kinds of progeny observed when the duplication-deficiency plants are used as seed parents and are pollinated by normal blandina are recorded in table 4 . This shows the kinds of gamete which can survive through the ovules and function in the formation of viable gametes. It demonstrates also, what has been shown previously (Catcheside, 1947a), that there is strong competition between gametes in the formation of embryo sacs. Thus $3 \cdot 4+3 \cdot 1 \mathrm{I}$ is at a serious disadvantage in competition with the normal $3.4+I I \cdot I 2$. In this

TABLE 4

Progeny of duplication-deficiency heterozygotes pollinated by normal plants. The zygotes would have $3 \cdot 4+11 \cdot 12$ from the normal parent

\begin{tabular}{|l|c||c|c|}
\hline Gramete from $A-D p$ & Progeny & Gamete from $A-D f$ & Progeny \\
\hline & & & \\
$3 \cdot 4+11 \cdot 12$ & 1035 & $3 \cdot 4+11 \cdot 12$ & 271 \\
$3 \cdot 4+3 \cdot 11$ & 108 & $4 \cdot 12+11 \cdot 12$ & 0 \\
$3 \cdot 4+3 \cdot 4+11 \cdot 12$ & 2 & $3 \cdot 4+11 \cdot 12+4 \cdot 12$ & 12 \\
$3 \cdot 4+3 \cdot 11+11 \cdot 12$ & $1 ?$ & & 283 \\
& $-\frac{1146}{114}$ & & \\
\hline
\end{tabular}

competition it has a bare ten per cent. chance of success. Moreover, the duplication-deficiency gamete of $A-D f$, namely $4 \cdot 12+11 \cdot 12$, appears to have no chance at all against the normal.

Turning to gametes with an extra chromosome, as evidenced by their trisomic progeny, there are very great differences in relative viability, though it is not known in what ways this takes effect. Generally, the gamete with an extra chromosome would compete with a gamete deficient for a chromosome and so be unlikely to be prevented by that cause from forming embryo sacs. It is more likely that the different balances of chromosomes may affect rates of development and so the chance of formation of a viable zygote. In practice, the likely type of trisomic occurs quite commonly from $A-D f$ and hardly at all, if at all, from $A-D p$. The one possible case of a tertiary trisomic with $3 \cdot 1$, from an $A-D p$ seed parent, is not certainly established, as the cytological evidence was unsatisfactory. The only trisomics certainly identified in $A-D p$ progeny have been the two 3.4 primary trisomics (" whitish"). They appear to have arisen more frequently than would be expected from primary non-disjunction in the normal diploid. Perhaps the frequency of non-disjunction is affected by the presence of the interchange chromosome. 
(v) Other duplication-deficiency progeny of interchange heterozygotes

Recently, two progenies of interchange heterozygotes have been grown with the object of maintaining the stock. It happened that one of them was segregating for the marker $n$ (nanella, a dwarf habit of growth) situated on chromosome arm 4. Both progenies contained a few exceptional plants which may represent products of the expected, but hitherto unobserved, non-disjunctional gametes. Unfortunately, for reasons which will appear, no cytological observations could be made to test these possibilities.

One progeny, of 12 I plants, was from the cross $\frac{A P^{r e}}{+P^{s}} \times+P^{s}$ and included $5^{6}+P^{s}, 5^{8} A P^{r e}$ and $2 A P^{s e}$. There were also a subwhitish trisomic with $P^{s}$ buds and a probable $A-D p P r$, which did not flower, so that the identification is uncertain. The remaining three plants were of two new types. One (type X) resembled the interchange heterozygote, but was more slender and differed in foliage characters; the plants, two in number, carried $P^{r}$ and showed a slight variegation of $P^{r}$, but generally the buds were shed prior to meiosis. The other (type Y) more nearly resembled normal blandina, but was rather more vigorous and lush, with larger arched leaves. The flowers were most extraordinary. They had long ovaries, each with a slight stalk at the base, very short hypanthia and short buds. The anthers were thin, never dehisced and generally turned brown before maturity. The styles were thin and bore small stigmas. No seeds were produced. Later in the season, the buds abruptly changed to having foliar petals, stamens and stigmas and these phyllodic flowers persisted open on the plant until it died. No satisfactory cytological material was obtained from either of these new kinds of progeny.

The second progeny, of 122 plants, was from the cross

$$
\frac{A+P^{r e}}{+n P^{s}} \times+n P^{s} \text {. }
$$

The parentage is similar to the previous progeny, except for heterozygosity in the seed parent for nanella $(n)$, located in chromosome arm 4. The regular progeny included $39+n P^{s}, \mathrm{I}++P^{s}, \mathrm{I}+n P^{r}, 70 A+P^{r e}$, $4 A n P^{r e}$ and I $A+P^{s e}$. The remaining six plants were of four types. One was a typical $A-D f$ plant with green buds and tall habit. A second was probably $A-D p$ and had $P^{r e r}$ buds and a nanella habit. The statures of these two plants agree with expectation. A third was similar to the type $\mathrm{X}$ in the previous progeny, had foliage darker than is usual for the interchange heterozygote and nearly all of the buds were shed when quite small. The remaining three plants were similar to the type Y in the previous progeny, except that all of them were nanella in habit; their flowers were predominantly of the phyllodic sort.

It is possible that the plants of types $\mathrm{X}$ and $\mathrm{Y}$ represent the two classes of progeny resulting from the $3 \cdot 4+4 \cdot 12$ and II I $2+3 \cdot 1$ I duplication-deficiency gametes, not previously detected. The absence 
of cytological data, due to difficulties of the plants themselves, prevents a completely critical test. However, several of the facts about the phenotypes of the plants agree with the suggestion that $3 \cdot 4+4 \cdot 12$ corresponds to type $\mathrm{Y}$ and II I $2+3 \cdot \mathrm{I}$ i to type $\mathrm{X}$. From the heterozygote $\frac{P^{r} 3 \cdot 11+n^{+} 4 \cdot 12}{P^{s} 3 \cdot 4 n+11 \cdot 12}$ crossed to $P^{s} 3 \cdot 4 n+1$ I.12 we would expect type $\mathrm{X}$ to show $P^{r}$ variegation, to be generally similar to the interchange hetcrozygote and to be nanella since the duplication-deficiency gamete would completely lack arm 4 in which $n$ is located. However, the possible instance in the second progeny was not a dwarf plant. Type $\mathrm{Y}$ would be expected to be $P^{s}$ in bud type, to be generally more similar to normal blandina in habit and not to be nanella since the duplication-deficiency gamete would be $P^{s} 3 \cdot 4 n+n^{+} 4 \cdot{ }^{-1} 2$ and the zygote would therefore be $n n n^{+}$. However, it is possible that the unexpectedly dwarf habit of the plants in the second progeny is due to the unbalanced chromosome set affecting the expression. The tentative identifications must therefore be treated with caution.

\section{SUMMARY}

An interchange heterozygote, involving two chromosomes and therefore showing a ring of four chromosomes at meiosis, is potentially capable of producing fourteen types of gamete containing one, two or three of these chromosomes. There are (i) the two regularly balanced gametes, normal and interchange, resulting from alternate disjunction, (ii) the four duplication-deficiency gametes resulting from adjacent disjunction, (iii) the four extra chromosome gametes resulting from numerical non-disjunction and (iv) the four gametes, each lacking a whole chromosome, also the result of numerical non-disjunction. In the case of the interchange in Enothera blandina, which shows a position effect variegation of the genes $P$ and $S$, most of the first three classes will produce viable progeny when combined with a normal hblandina gamete. The correlations correct the previous interpretation of certain duplication-deficiency plants.

\section{REFERENCES}

CAtcheside, D. G. 1940. Structural analysis of CEnothera complexes. Proc. Roy. Soc. Lond., B, I28, 509-535.

Catcheside, D. G. 1947a. The P-locus position effect in Enothera. 7. Genet., $4^{8}$, $31-42$.

CATCHESide, D. G. 1947b. A duplication and a deficiency in CEnothera. 7. Genet., 48 . 99-IIO.

Catcheside, D. G. 1954. The genetics of brevistylis in Enothera. Heredity, 8, 125-137. goldschmidt, R. 1958. Genic conversion in Enothera? A critical review. Amer. Nat., 92, 93-104. 